ITC 2/47

Journal of Information Technology and Control

Vol. 47 / No. 2 / 2018

pp. 295-309

DOI 10.5755/j01.itc.47.2.16288

(c) Kaunas University of Technology
A Hybrid Optimal Controller Based on the Robust Decoupled Sliding Mode and Adaptive Feedback Linearization

Received 2016/09/24

Accepted after revision 2018/03/13

\title{
A Hybrid Optimal Controller Based on the Robust Decoupled Sliding Mode and Adaptive Feedback Linearization
}

\section{J. Mahmoodabadi, T. Soleymani}

Department of Mechanical Engineering, Sirjan University of Technology, Sirjan, Iran

\section{Andalib Sahnehsaraei}

Iranian Gas Transmission Co., National Iranian Gas Company (NIGC), Ramsar, Iran

Corresponding author: mahmoodabadi@sirjantech.ac.ir

In this paper, a robust adaptive control method for a class of fourth-order systems is proposed. The used structure for this controller is a combination of decoupled sliding mode approach and feedback linearization technique. The decoupled sliding mode is applied to guarantee the sliding condition, and by applying the feedback linearization method, a linear control law with adaptive coefficients is employed. The final control effort is defined as the weighting summation of the decoupled sliding mode and feedback linearization controllers. Then, the controller coefficients are optimized using the multi-objective genetic algorithm. Finally, to show effectiveness of the proposed approach, it is applied to handle the cart-pole, ball-beam, and ball-wheel systems and the results are compared with those reported in the literature.

KEYWORDS: Feedback linearization, Decoupled sliding mode, Robust adaptive control, Multi-objective genetic algorithm.

\section{Introduction}

Over the past few years, control of nonlinear systems with uncertainties as a pivotal problem in the field of control engineering has been intensively studied by researchers. One of the most popular methods widely applied to handle uncertainties and external disturbances is sliding mode control (SMC) [13]. This 
method has major advantages, such as guaranteed stability, robustness against parametric variations, fast dynamic response, and ease of implementation. In the sliding-mode control theory, control dynamics have two sequential modes $[13,15]$. The first is the reaching mode and the second is the sliding mode. At the first stage, a parameter, called sliding surface, is defined as a weighting summation of error and at the second stage, a control law is designed in order to push the states toward the sliding surface. Mahmoodabadi et al. proposed an optimal robust sliding mode tracking controller for a biped robot [11]. Unlike the SMC technique which can be applied to the systems with the canonical form only, Decoupled Sliding Mode Control (DSMC) is suitable to control systems with the non-canonical forms. In DSMC, the fourth order systems are divided into two subsystems; then, the sliding surfaces are separately designed for each of them, and a control law which converts the state variables to the desired vector is designed. This control law can be used for both subsystems since the targets of the subsystems are achieved. In [6], the control of nonlinear systems based on DSMC with reliance on concepts of fuzzy-neural network is presented. Mahmoodabadi et al. introduce an online optimal control based on DSMC in order to decrease dependence of initial conditions on electromechanical systems [10]. Mahmoodabadi et al. presented an optimal control for the inverted pendulum system using DSMC [9]. In [2, 3], DSMC with time-varying coefficients for sliding surfaces is investigated in which the coefficients are computed based on fuzzy rules.

Another way to control uncertain systems with known dynamic structure, but unknown constant or slowly-varying parameters is adaptive control [13]. This type of controller usually includes a control law whose parameters are adjusted based on a suitable mechanism, and the most important part in such a controller is tuning mode of the control parameters. In [12], an adaptive robust PID control subject to supervisory decoupled sliding mode control is proposed for an inverted pendulum and cart system. In [14], an adaptive robust hybrid of PID and sliding control is designed for a biped robot.

In addition, much attention always have been paid to the linearization of nonlinear systems and use of a linear controller by researchers. The method of Jacobean linearization equations has been used for many years, but this method is applicable for small range around the equilibrium point. Feedback linearization (FBL), as one of nonlinear control methods, uses the method of changing variables instead of approximating variables, and is applicable to a wider range in comparison with the Jacobean method [7, 13]. In [4], an FBL method is approximately applied to a ball-beam system, and [5] uses the FBL method to control of a ball-wheel system. In [1], a combination of FBL and DSMC is implemented to control a cart-pole system.

In the present paper, a control law based on the weighting sum of the FBL and DSMC methods is proposed, as the main idea of this is to enhance efficiency and robustness against uncertainties. First, FBL and DSMC are simultaneously implemented; then, the coefficients of FBL controller are set based on an approximate gradient descend method so that the sliding condition in DSMC method can be met. Thereafter, the control parameters are obtained using the multi-objective genetic algorithm optimization (MOGA) so that the integral of time multiplied by absolute error is minimized. The good advantage of this work that plays the key role in the control field is to have a series of optimum points as Pareto front.

The paper is organized in five sections. Section 2 reviews the DSMC and FBL methods. The proposed control strategy is presented in Section 3. In Section 4 , numerical simulation of the proposed method is given for three popular systems in control laboratory setting compared with conventional methods in other articles. Finally, conclusions are provided in Section 5.

\section{Decoupled Sliding Mode and Feedback Linearization Controllers}

Consider a fourth order under actuated nonlinear system in the non-canonical form as follows:

$\dot{x}_{1}(t)=x_{2}(t)$
$\dot{x}_{2}(t)=f_{1}(\mathbf{x})+g_{1}(\mathbf{x}) u(t)+d_{1}(t)$
$\dot{x}_{3}(t)=x_{4}(t)$
$\dot{x}_{4}(t)=f_{2}(\mathbf{x})+g_{2}(\mathbf{x}) u(t)+d_{2}(t)$


where $\mathbf{x}(t)=\left[x_{1}(t), x_{2}(t), x_{3}(t), x_{4}(t)\right]^{\mathrm{T}}$ denotes the state vector, $f_{1}(\mathbf{x}), f_{2}(\mathbf{x}), g_{1}(\mathbf{x})$, and $g_{2}(\mathbf{x})$ are nonlinear functions, functions, $u(t)$ is the control input, and $d_{1}(t)$ and $d_{2}(t)$ are external disturbances. The disturbances are assumed to be bounded as $\left|d_{1}(t)\right| \leq D_{1}(t)$ and $\left|d_{2}(t)\right| \leq D_{2}(t)$.

\subsection{Decoupled Sliding Mode Control (DSMC)}

The main idea behind decoupled sliding mode control is to design a control law in a way that several subsystems are simultaneously controlled using only one input [10]. For the fourth order systems, the state variables $x_{1}$ and $x_{2}$ are chosen to form the subsystem A (primary target) and the state variables $x_{3}$ and $x_{4}$ are used to form the subsystem B (secondary target), and the following sliding surfaces are defined:

$\mathrm{s}_{1}=\mathrm{c}_{1}\left(x_{1}(t)-z\right)+x_{2}(t)$
$\mathrm{s}_{2}=\mathrm{c}_{2} x_{3}(t)+x_{4}(t)$

where $c_{1}$ and $c_{2}$ are strictly positive design parameters and $z$ is a mediator variable of a decaying oscillation signal whose value is dependent on $s_{2}$, and can be expressed as follows:

$$
z=\operatorname{sat}\left(\frac{s_{2}}{\emptyset_{z}}\right) z_{\text {upper }},
$$

where, $z_{\text {upper }}$ transfers $s_{2}$ to the proper range of $x_{1}(t)$ :

$$
|z| \leq z_{\text {upper }} ; 0<z_{\text {upper }}<1 .
$$

It is ensured that $x_{1}(t)$ is finite if Equation (8) holds. Moreover, $\emptyset_{z}$ is the boundary layer of $s_{2}$ and transfers $s_{2}$ to a proper range of $x_{1}(t)$. Function sat is also defined as below:

$$
\operatorname{sat}(\varnothing)=\left\{\begin{array}{ll}
\operatorname{sgn}(\varnothing) & |\varnothing| \geq 1 \\
\varnothing & |\varnothing| \leq 1
\end{array} .\right.
$$

Equation (5) means the control objective $u(t)$ is changed from $x_{1}(t)=0, x_{2}(t)=0$ to $x_{1}(t)=z, x_{2}(t)=0$. To ensure the stability of the system, the control effort should be defined as follows:

$$
u_{D S M C}(t)=u_{e q}(t)-\operatorname{sat}\left(s_{1} G\right) P_{S},
$$

where $P_{s}$ and $G$ are design parameters and $u_{e q}(t)$ is the equivalent control input obtained by using $\dot{s}_{1}=0$ as follows:

$$
u_{e q}(t)=\frac{1}{g_{1}}\left(c_{1}\left(\dot{z}(t)-x_{2}(t)\right)-f_{1}(\mathbf{x})\right) .
$$

$u_{e q}(t)$ keeps the states on the sliding surface and non-continuous statement moves the states toward sliding surface.

\subsection{Feedback Linearization (FBL)}

Suppose the governing equations are according to Equations (1) to (4). Feedback linearization is done in two ways, input-states and input-output. For input-state linearization, one must check controllability conditions on $\left\{g, a d_{f} g, \ldots, a d_{f}^{n-1} g\right\}$ and involutivity conditions on $\left\{g, a d_{f} g, \ldots, a d_{f}^{n-2} g\right\}[7,13]$. If the conditions are satisfied, $\zeta_{1}$ could be derived as the first linear dynamics from the following equations:

$$
\nabla \zeta_{1} a d_{f}^{i} g=0 \quad i=0, \ldots, n-2
$$

$\nabla \zeta_{1} a d_{f}^{n-1} g \neq 0$,

where $a d_{f}^{i} g$ denotes Lie brackets $f$ and $g . n$ is the order of the dynamic equations. Other states can be obtained from the following transformation:

$$
\zeta(\mathbf{x})=\left[\zeta_{1}, l_{f} \zeta_{1}, \ldots, l_{f}^{n-1} \zeta_{1}\right]^{T},
$$

where $l_{f} \zeta_{1}$ signifies Lie derivative of $\zeta_{1}$ with respect to $f$. Finally, the input transformation is defined as the following.

$$
u_{F B L}(t)=\alpha(\mathbf{x})+\beta(\mathbf{x}) v
$$

where

$$
\begin{aligned}
& \alpha(\mathbf{x})=-\frac{l_{f}^{n} \zeta_{1}}{l_{g} l_{f}^{n-1} \zeta_{1}} \\
& \beta(\mathbf{x})=\frac{1}{l_{g} l_{f}^{n-1} \zeta_{1}},
\end{aligned}
$$

where $\boldsymbol{v}$ is a linear control input as below:

$$
v=-k_{1} \zeta_{1}-k_{2} \zeta_{2}-k_{3} \zeta_{3}-k_{4} \zeta_{4},
$$

where $k_{1}, k_{2}, k_{3}$, and $k_{4}$ are constant and chosen to take the poles of the closed loop systems in the left imaginary axes.

The basic procedure of input-output linearization is to derive the output function repeatedly until the in- 
put appears, and then is designed to cancel the nonlinearity $[7,13]$.

\section{The Proposed Controller}

In this paper, the proposed controller is a linear combination of DSMC and FBL methods according to the following equation:

$$
u(t)=c_{S} \times u_{D S M C}(t)+c_{f} \times u_{F B L}(t)
$$

where $c_{s}$ and $c_{f}$ are weighting coefficients. Furthermore, the control coefficients $k_{1}, k_{2}, k_{3}$, and $k_{4}$ of the FBL method are adjusted using an approximate gradient descent method according to the following:

$$
\dot{\mathrm{k}}_{\mathrm{i}}=-\gamma_{\mathrm{i}} \frac{\partial \mathrm{s}_{1} \dot{\mathrm{s}}_{1}}{\partial \mathrm{k}_{1}} \equiv-\gamma_{\mathrm{i}} \mathrm{s}_{1} \zeta_{\mathrm{i}} \quad i=1,2,3,4,
$$

where $\gamma_{i}$ is a positive constant, and $\zeta_{i}$ is the new state which is derived after the applied FBL method.

The proposed strategy, a weighting summation of DSMC and adaptive FBL controller, is abbreviated as SDSAF, and Fig.1 provides its graphical representation. In this figure, the performance evaluation block calculates the value of the objective function defined as the integral of time of the absolute errors (ITAE) that must be minimized:

$$
\operatorname{ITAE}=\int t|e(t)| d t
$$

where $e(t)$ depicts the error signal between the desired and output states..

In addition, two constraints for the control effort are considered as follows:

$\frac{\max |u(t)|<m_{u}}{\int u(t) d t<s_{u}}$

where $m_{u}$ and $s_{u}$ are, respectively, maximum control input and maximum integral of the control input.

Furthermore, the multi-objective genetic algorithm (MOGA) optimization block is applied to select the control parameters, introduced in the first column of Table 2 as one of the most important parts in the design procedure of controllers. The specifications of the genetic algorithm are as: population size 200, tournament selection function, crossover fraction 0.8, Pareto front population fraction 0.35 and maximum generation 5000 .

\section{Figure 1}

Graphical representation of the SDSAF controller

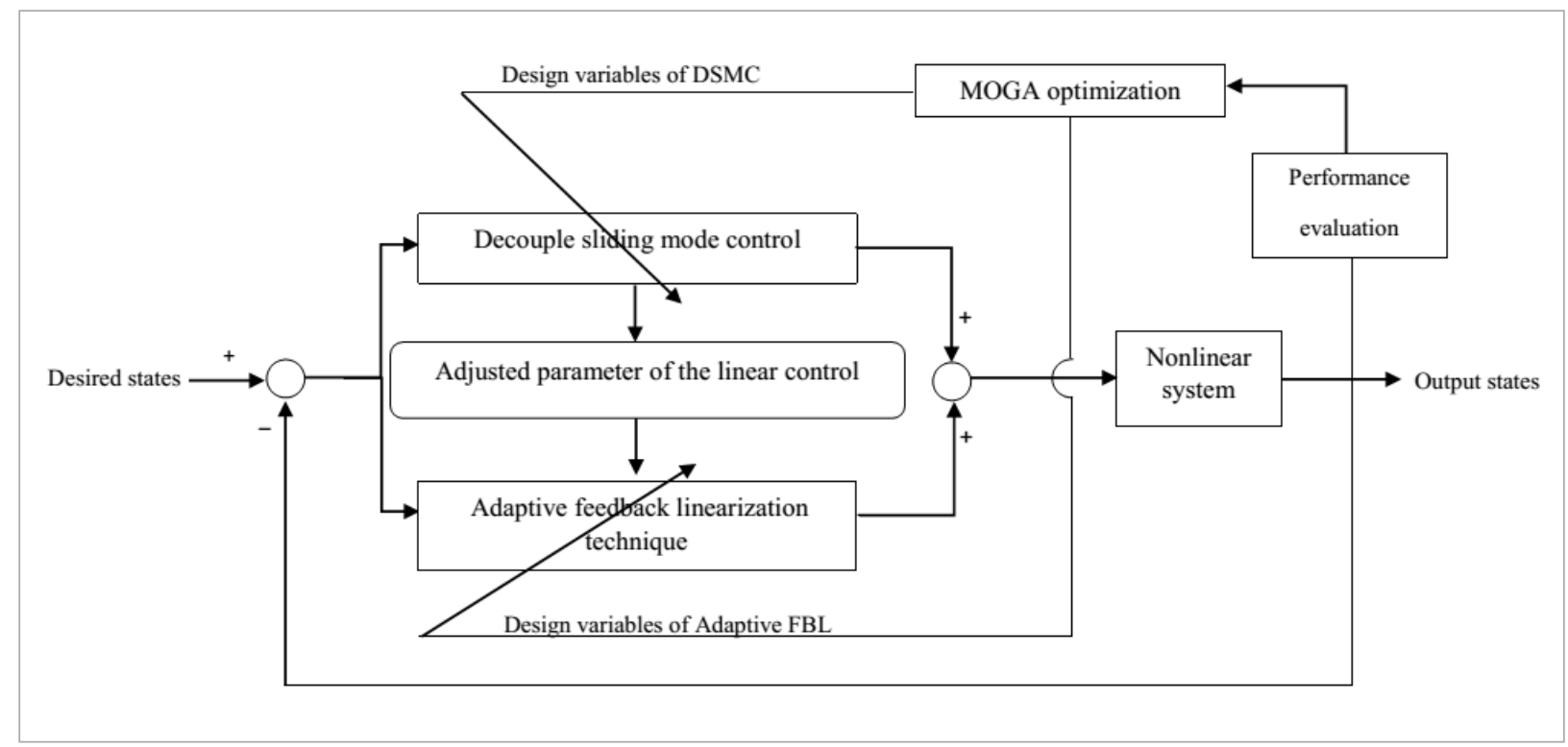


Figure 2

The physical configuration of the cart-pole system

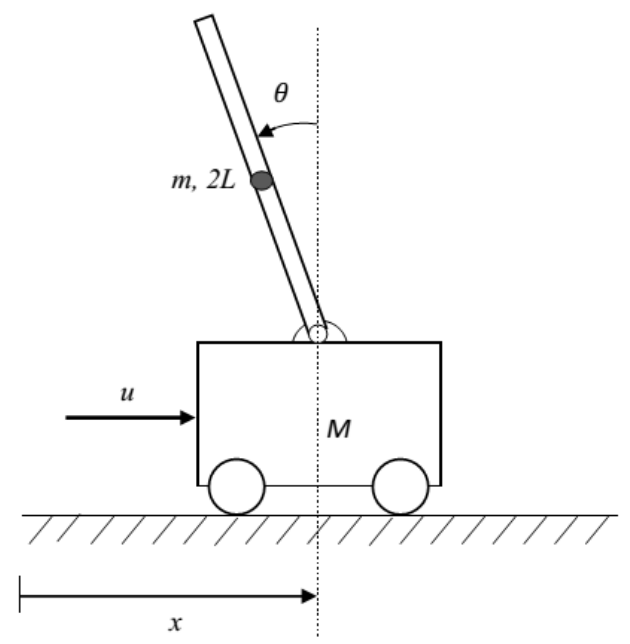

\section{Simulation Results}

In this section, the ability of the proposed controller is challenged to control three nonlinear and under- actuated mechanical systems cart-pole, ball-beam, and ball-wheel system. For this, Pareto fronts and time responses by applying several methods are derived and are compared with each other.

\subsection{Cart-pole}

Fig. 2 shows the cart-pole system with variables $\left[x_{1}(t), x_{2}(t), x_{3}(t), x_{4}(t)\right]=[\theta(t), \dot{\theta}(t), x(t), \dot{x}(\mathrm{t})]$. Dynamical equations are expressed as follows:

$$
\begin{aligned}
& \dot{x}_{1}(t)=x_{2}(t) \\
& \dot{x}_{2}(t)=\frac{(M+m) g \sin x_{1}(t)-m L \sin x_{1}(t) \cos x_{1}(t) x_{2}^{2}(t)}{L\left(\frac{4}{3}(M+m)-m \cos ^{2} x_{1}(t)\right)}+ \\
& +\frac{\cos x_{1}(t)}{L\left(\frac{4}{3}(M+m)-m \cos ^{2} x_{1}(t)\right)} u(t)+d \\
& \dot{x}_{3}(t)=x_{4}(t) \\
& \dot{x}_{4}(t)=\frac{m g \sin x_{1}(t) \cos x_{1}(t)-\frac{4}{3} m L x_{2}^{2}(t) \sin x_{1}(t)}{\frac{4}{3}(M+m)-m \cos ^{2} x_{1}(t)}+ \\
& +\frac{\frac{4}{3}}{\frac{4}{3}(M+m)-m \cos ^{2} x_{1}(t)} u(t)+d .
\end{aligned}
$$

Physical specifications of the system as well as the initial values are assumed to be: $M=1 \mathrm{~kg}, \mathrm{~m}=0.05 \mathrm{~kg}$, $\mathrm{L}=0.5 \mathrm{~m},|d| \leq 0.08, g=9.8 \frac{\mathrm{m}}{\mathrm{s}^{2}}$, and $\theta(0)=-60^{\circ}, \dot{\theta}(0)=0$, $x(0)=0, \dot{x}(0)=0$.

To achieve $u_{D S M C}(t)$ according to Equation (10), the sliding surface is calculated by the use of Equations (5) and (6):

$$
\begin{aligned}
& u_{D S M C}(t)=\frac{L\left(\frac{4}{3}(M+m)-m \cos ^{2} x_{1}(t)\right)}{\cos x_{1}(t)}\left(c_{1}\left(\dot{z}-x_{2}(t)\right)\right. \\
& \left.-\frac{(M+m) g \sin x_{1}(t)-m L \sin x_{1}(t) \cos x_{1}(t) x_{2}^{2}(t)}{L\left(\frac{4}{3}(M+m)-m \cos ^{2} x_{1}(t)\right)}\right)-P_{S} \operatorname{sat}\left(s_{1} G\right) .
\end{aligned}
$$

For implementation of FBL methods, first, the following nonlinear transformation is considered [1]:

$$
\begin{aligned}
& u(t)=\left((M+m)-\frac{3}{4} m \cos ^{2} x_{1}(t)\right) u(t)+ \\
& +m L x_{2}^{2}(t) \sin x_{1}(t)-\frac{3}{4} m g \sin x_{1}(t) \cos x_{1}(t) .
\end{aligned}
$$

Equations of motion can be rewritten as follows:

$\dot{\mathbf{x}}=\left[\begin{array}{c}x_{2} \\ \frac{3}{4 L} g \sin x_{1}(t) \\ x_{4}(t) \\ 0\end{array}\right]+\left[\begin{array}{c}0 \\ \frac{3}{4 L} \cos x_{1}(t) \\ 0 \\ 1\end{array}\right] u(t)=f(\mathbf{x})+g(\mathbf{x}) u(t)$

As involutivity condition is not satisfied, we use approximation linearization by a new state as $\zeta_{1}=\varnothing(\mathbf{x})=$ $x_{3}(t)-\frac{4 L}{3} \ln \left(\frac{1+\sin x_{1}(t)}{\cos x_{1}(t)}\right)[1]$; then other states would be obtained as follows:

$$
\dot{\zeta}_{1}=\zeta_{2}=L_{f} \emptyset(\mathbf{x})=x_{4}(t)-\frac{4 L}{3}\left(\frac{x_{2}(\mathrm{t})}{\cos x_{1}(t)}\right)
$$

$$
\dot{\zeta}_{2}=\zeta_{3}=L_{f}^{2} \emptyset(\mathbf{x})=-\tan x_{1}(t)\left(g+\frac{4 L x_{2}^{2}(t)}{3 \cos x_{1}(t)}\right)
$$

$$
\dot{\zeta}_{3}=\zeta_{4}=L_{f}^{3} \emptyset(\mathbf{x})=-\frac{4 L}{3}\left(\frac{2}{\cos ^{3} x_{1}(t)}-\frac{1}{\cos x_{1}(t)}\right) x_{2}^{3}(t)-
$$$$
-\left(\frac{3 g}{\cos ^{2} x_{1}(t)}-2 g\right) x_{2}(t)-2 x_{2}(t) \tan x_{1}(t) \dot{u}(t) \text {. }
$$

By neglecting term $2 x_{2}(t) \tan x_{1}(t) \dot{u}(t)$, then

$$
\dot{\zeta}_{4}=L_{f}^{4} \emptyset(\mathbf{x})+L_{g} L_{f}^{3} \emptyset(\mathbf{x}) \dot{u}(t)
$$




$$
\begin{aligned}
& L_{f}^{4} \emptyset(\mathbf{x})=-\frac{4 L}{3}\left(\frac{\sin x_{1}(t)}{\cos ^{2} x_{1}(t)}\right)\left(\frac{6}{\cos ^{2} x_{1}(t)}-1\right) x_{2}^{4}(t)- \\
& \left(\frac{3 g \sin x_{1}(t)}{\cos x_{1}(t)}\right)\left(\frac{4}{\cos ^{2} x_{1}(t)}-1\right) x_{2}^{2}(t)+ \\
& \frac{3 g}{4 L} \sin x_{1}(t)\left(2 g-\frac{3 g}{\cos ^{2} x_{1}(t)}\right)
\end{aligned}
$$

$L_{g} L_{f}^{3} \emptyset(\mathbf{x})=3 x_{2}^{2}(t)-\frac{6 x_{2}^{2}(t)}{\cos ^{2} x_{1}(t)}-\frac{9 g}{4 L \cos x_{1}(t)}+\frac{3 g}{2 L} \cos x_{1}(t)$.

The control input is given by

$$
\dot{u}_{F B L}(t)=\frac{v-L_{f}^{4} \emptyset(\mathbf{x})}{L_{g} L_{f}^{3} \emptyset(\mathbf{x})},
$$

where $v$ is given by Equation (18), and thus $u_{F B L}(t)$ is given by Equations (37) and (29).

After applying the multi-objective optimization algorithm to this problem, the Pareto fronts of the angle error of the pole and distance error of the cart demonstrated in Fig. 3 would be achieved.

The value of optimization constraints and objective functions corresponding to the optimum design points, A, B, and C in Pareto front and their design variables are shown in Tables 1 and 2, respectively. The results in Fig. 3 and Table 1 show that not only the proposed SDSAF satisfies the limitations better than the FBL and DSMC methods, but it also has the smallest value of the objective functions.

Figure 3

The obtained Pareto fronts by using MATLAB's Toolbox MOGA for FBL, DSMC and SDSAF for the cart-pole system

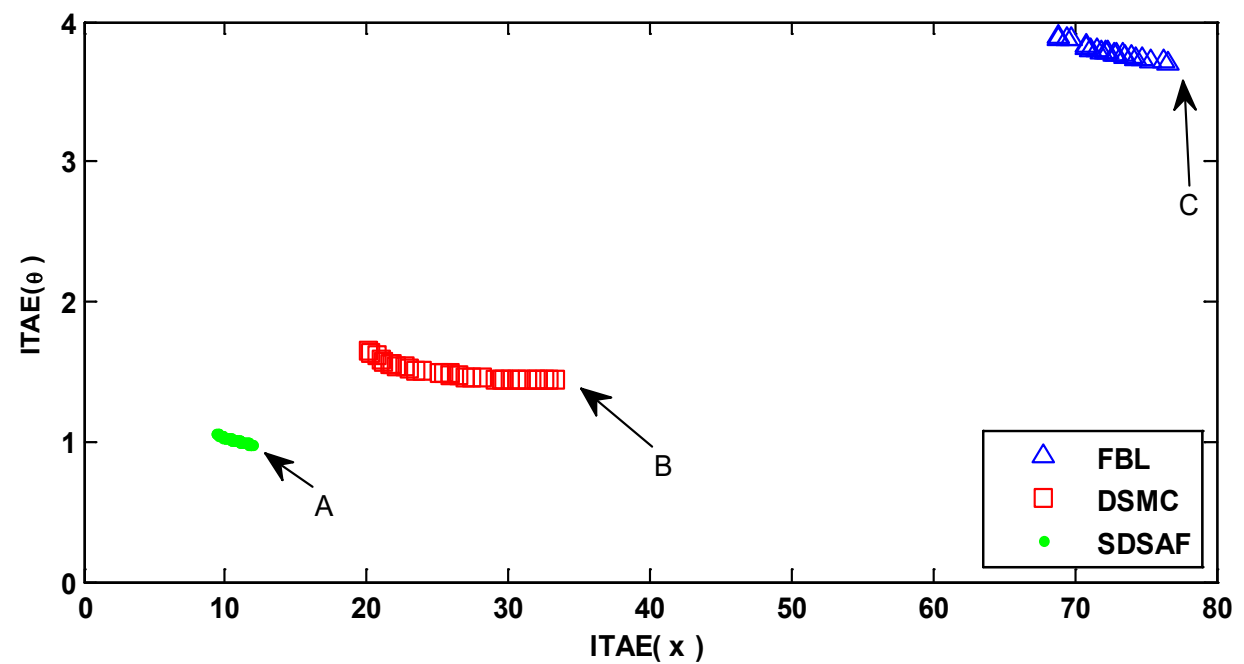

Table 1

Values of optimization constraints and objective functions for the optimum points A, B, and C of Fig. 3

\begin{tabular}{l|c|c|c}
\hline & FBL (point C) & DSMC (point B) & SDSAF (point A) \\
\hline $\mathbf{m}_{\mathrm{u}}$ & 150 & 150 & 130 \\
\hline $\mathbf{S}_{\mathrm{u}}$ & 55 & 32 & 30 \\
\hline ITAE $(\boldsymbol{\theta})$ & 3.888 & 1.436 & 0.9635 \\
\hline ITAE (x) & 68.85 & 33.26 & 12.09
\end{tabular}


The time responses of these optimum design points and obtained results in Ref. [8] are compared in Figs. 4 and 5. Furthermore, their control inputs are illustrated in Fig. 6. As a result, Figs. 4, 5, and 6 conspicuously show that the SDSAF method exhibits a faster response than the other methods while its maximum overshoots are less than the others. In addition, it is clear that the SDSAF control method can hold the cart in a shorter distance, which confirms the fact that it is quicker than the other methods.

Table 2

Design variables for the optimum points A, B, and C of Fig. 3

\begin{tabular}{l|c|c|c}
\hline & $\begin{array}{c}\text { FBL } \\
\text { (point C) }\end{array}$ & $\begin{array}{c}\text { DSMC } \\
\text { (point B) }\end{array}$ & $\begin{array}{c}\text { SDSAF } \\
\text { (point A) }\end{array}$ \\
\hline$c_{1}$ & - & 4.2 & 6.15 \\
\hline$c_{2}$ & - & 0.4 & 0.53 \\
\hline$\emptyset_{\mathbf{z}}$ & - & 8.2 & 10.72 \\
\hline $\mathbf{Z}_{\text {upper }}$ & - & 0.999 & 0.9437 \\
\hline$\gamma_{1}, \mathbf{k}_{1}$ & 28.15 & - & 74.21 \\
\hline$\gamma_{2}, \mathbf{k}_{2}$ & 26.34 & - & 110.3 \\
\hline$\gamma_{3}, \mathbf{k}_{3}$ & 38.9 & - & 0.0695 \\
\hline$\gamma_{4}, \mathbf{k}_{4}$ & 6.15 & - & 0.0014 \\
\hline
\end{tabular}

\begin{tabular}{l|c|c|c}
\hline & $\begin{array}{c}\text { FBL } \\
\text { (point C) }\end{array}$ & $\begin{array}{c}\text { DSMC } \\
\text { (point B) }\end{array}$ & $\begin{array}{c}\text { SDSAF } \\
\text { (point A) }\end{array}$ \\
\hline $\boldsymbol{P}_{s}$ & - & 20.76 & 10.67 \\
\hline $\boldsymbol{G}$ & - & 0.86 & 0.59 \\
\hline $\mathbf{C}_{\mathbf{s}}$ & - & - & 0.97 \\
\hline $\mathbf{C}_{\mathbf{f}}$ & - & - & 1.3 \\
\hline $\mathbf{k}_{\mathbf{1}}(\mathbf{0})$ & - & - & 179.1 \\
\hline $\mathbf{k}_{2}(\mathbf{0})$ & - & - & 224.2 \\
\hline $\mathbf{k}_{3}(\mathbf{0})$ & - & - & 0.0007 \\
\hline $\mathbf{k}_{4}(\mathbf{0})$ & - & - & 0.0425 \\
\hline
\end{tabular}

Figure 4

Time responses of the pole angle corresponding to the optimum design points A, B, and C shown in the Pareto fronts and [8]

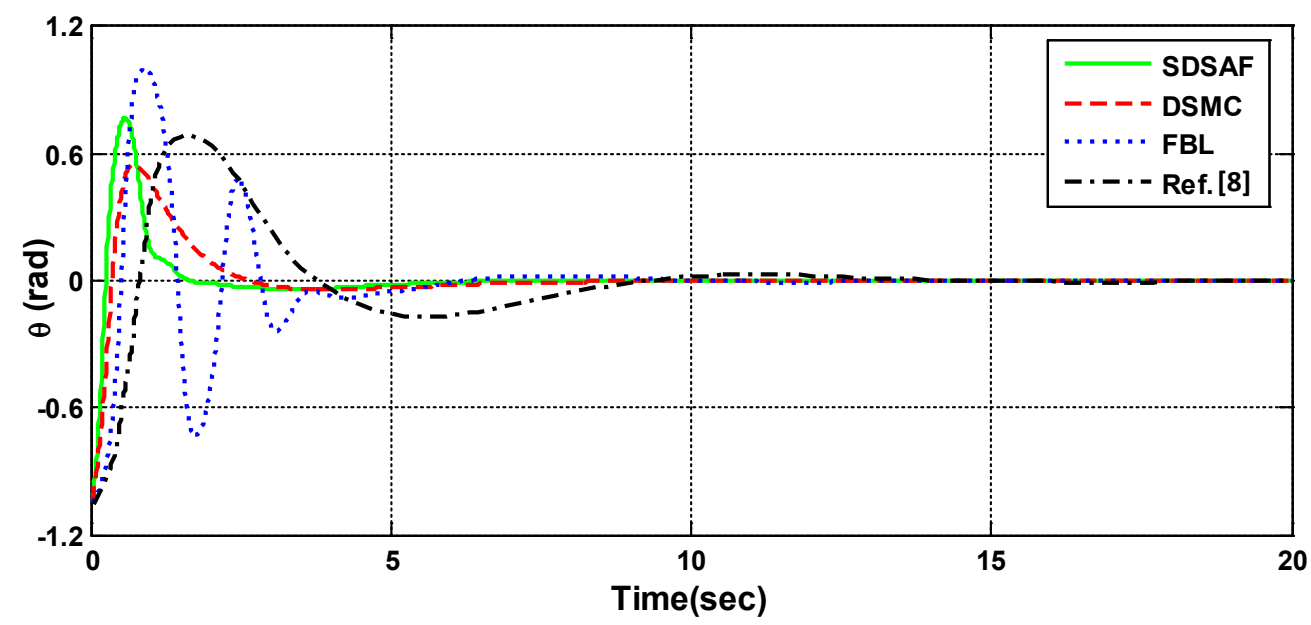


Figure 5

Time responses of the cart position corresponding to the optimum design points A, B, and C shown in the Pareto fronts and [8]

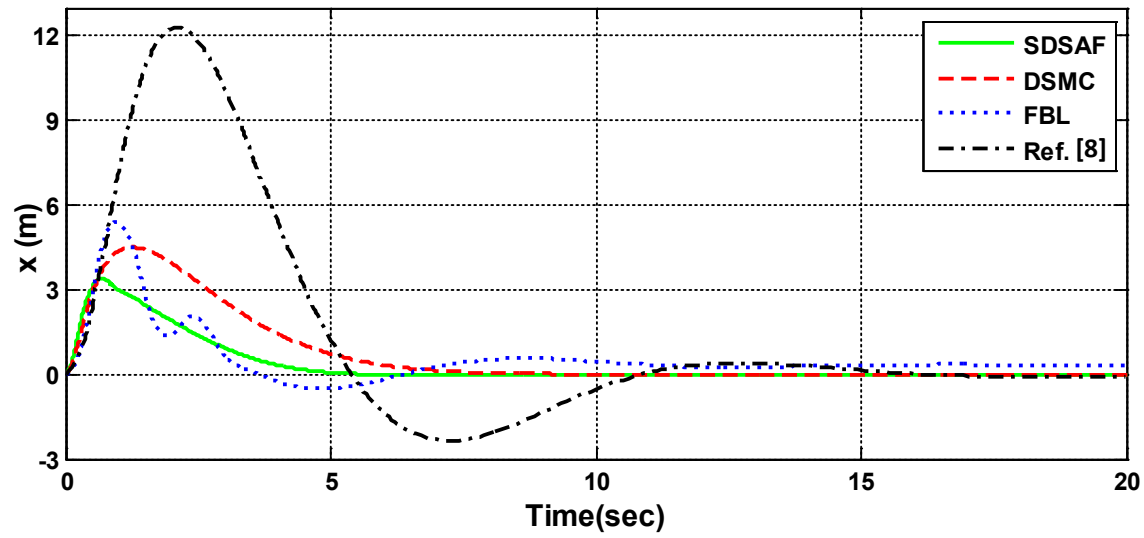

\section{Figure 6}

Control input of the inverted pedulum system corresponding to the optimum design points A, B, and C shown in the Pareto fronts and [8]

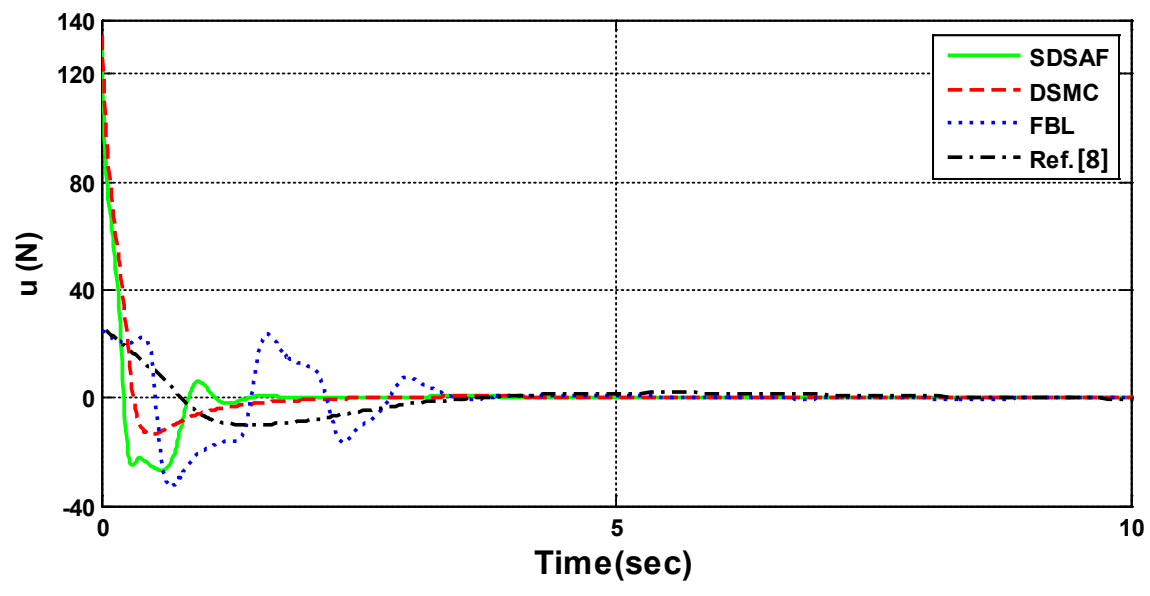

\subsection{Ball-beam}

For the Ball-beam system shown in Fig. 7 , the state space equations of motion are as follow with $\left[x_{1}(t)\right.$, $\left.\left.x_{2}(t), x_{3}(t), x_{4}(t)\right]=[\theta(t), \dot{\theta}(t), r(t), \dot{r}(\mathrm{t})]\right]$ :

$$
\dot{x}_{1}(t)=x_{2}(t)
$$

Figure 7

The physical configuration of the ball-beam system

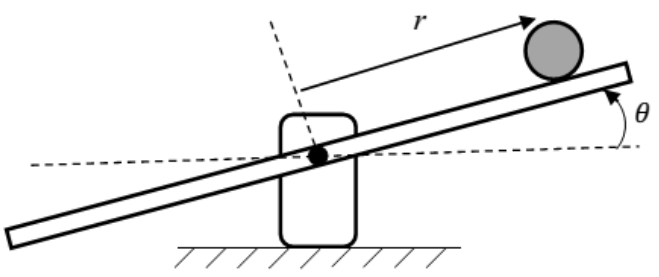


$\dot{x}_{2}(t)=u(t)+d(t)$

$\dot{x}_{3}(t)=x_{4}(t)$

$\dot{x}_{4}(t)=B\left(x_{3}(t) x_{2}^{2}(t)-g \sin x_{1}(t)\right)$,

where $B=\frac{M R^{2}}{J_{b}+M R^{2}}$ that $J_{b}, M$, and $R$ are, respectively, inertia moment of the ball, mass of the ball, and radius of the ball.

The above equations were obtained with simplifying assumptions such as frictionless rotation center and rotation without sliding of the ball on the beam.

Specifications used in the simulation study are $\mathrm{B}=0.7143, J_{b}=2 \times 10^{-6}, \mathrm{M}=0.05 \mathrm{~kg}, \mathrm{R}=0.01 \mathrm{~m}, \mathrm{~g}=9.8 \frac{\mathrm{m}}{\mathrm{s}^{2}}$, $|d| \leq 0.08$, and the initial values are $\theta(0)=60^{\circ}, \dot{\theta}(0) \stackrel{\mathrm{s}^{2}}{=}$, $r(0)=10, \dot{r}(0)=0$.

By choosing the sliding surfaces $s_{1}$ and $s_{2}$ according to (5), (6), $u_{D S M C}(t)$ is obtained as follow:

$u_{D S M C}(t)=u_{e q}(t)=c_{1}\left(\dot{z}-x_{2}(t)\right)-\operatorname{sat}\left(s_{1} G\right) P_{s}$.

For implementation of FBL method, the first state is chosen as $\zeta_{1}=\emptyset(\mathbf{x})=x_{3}(t)$ [8]; consequently, the other states would be obtained as follows:

$$
\dot{\zeta}_{1}=\zeta_{2}=L_{f} \emptyset(\mathbf{x})=x_{4}(t)
$$

$\dot{\zeta}_{2}=\zeta_{3}=L_{f}^{2} \emptyset(\mathbf{x})=B\left(x_{3}(t) x_{2}^{2}(t)-g \sin x_{1}(t)\right)$.

By neglecting term $B x_{3}(t) x_{2}^{2}(t)$, we obtain

$\dot{\zeta}_{3}=\zeta_{4}=L_{f}^{3} \emptyset(\mathbf{x})=-B g x_{2}(t) \cos x_{1}(t)$

$\dot{\zeta}_{4}=L_{f}^{4} \emptyset(\mathbf{x})+L_{g} L_{f}^{3} \emptyset(\mathbf{x}) u(t)=$

$B g x_{2}^{2}(t) \sin x_{1}(t)+\left(-B g \cos x_{1}(t)\right) u(t)$

$u_{F B L}(t)=\frac{v-B g x_{2}^{2}(t) \sin x_{1}(t)}{-B g \cos x_{1}(t)}$.

By applying the multi-objective optimization to three methods FBL, DSMC, and SDSAF, the Pareto front is gained like that illustrated in Fig.8. Moreover, the constraint values and objective functions, which are designated in Pareto fronts, are delineated in Table 3.

Table 3

Values of optimization constraints and objective functions at the optimum points A, B, and C of Fig. 8

\begin{tabular}{l|c|c|c}
\hline & $\begin{array}{c}\text { FBL } \\
\text { (point C) }\end{array}$ & $\begin{array}{c}\text { DSMC } \\
\text { (point B) }\end{array}$ & $\begin{array}{c}\text { SDSAF } \\
\text { (point A) }\end{array}$ \\
\hline $\mathbf{m}_{\mathrm{u}}$ & 5 & 5 & 5 \\
\hline $\mathbf{s}_{\mathrm{u}}$ & 2 & 3 & 2 \\
\hline ITAE $(\theta)$ & 2.67 & 2.75 & 2.62 \\
\hline ITAE (r) & 27.7 & 25.67 & 21.05
\end{tabular}

\section{Figure 8}

The obtained Pareto fronts by using MATLAB's Toolbox MOGA for FBL, DSMC, and SDSAF for the ball-beam system

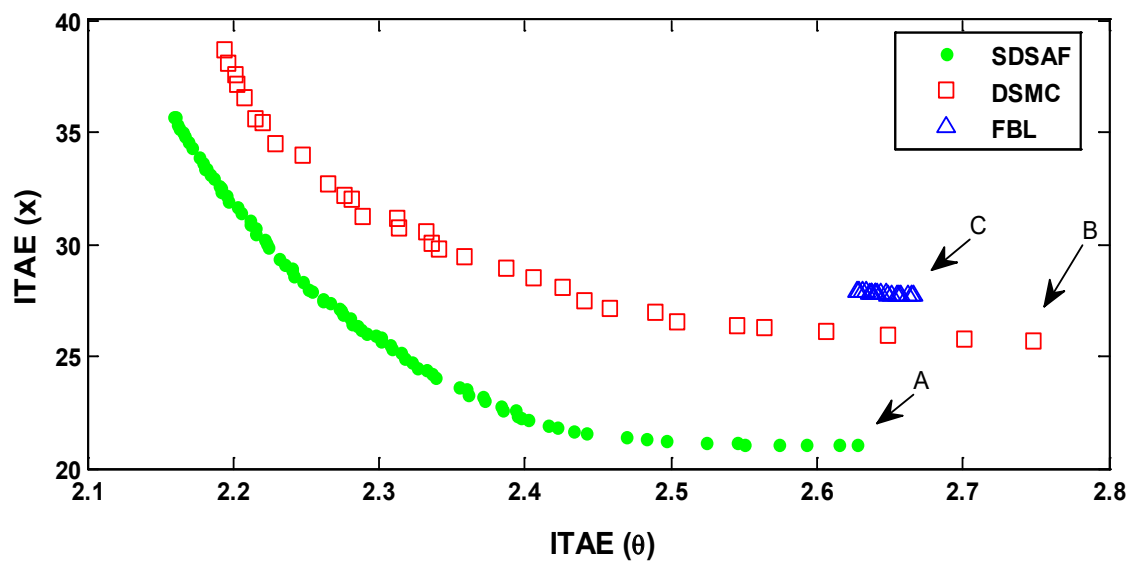


Clearly, the Pareto front achieved by the SDSAF method is better than other methods, though the value of their constraint has not distinction to the others. Table 4 represents design variables of points A, B, and $\mathrm{C}$ delineated in Pareto fronts of Fig. 8.

Table 4

Design variables for the optimum points A, B, and C of Fig. 8

\begin{tabular}{l|c|c|c}
\hline & $\begin{array}{c}\text { FBL } \\
\text { (point C) }\end{array}$ & $\begin{array}{c}\text { DSMC } \\
\text { (point B) }\end{array}$ & $\begin{array}{c}\text { SDSAF } \\
\text { (point A) }\end{array}$ \\
\hline $\mathbf{c}_{1}$ & - & 2.07 & 3.29 \\
\hline $\mathbf{c}_{2}$ & - & 0.54 & 0.63 \\
\hline$\emptyset_{\mathrm{z}}$ & - & 5.4 & 8.55 \\
\hline $\mathbf{Z}_{\text {upper }}$ & - & 0.999 & 0.977 \\
\hline$\gamma_{1}, \mathbf{k}_{1}$ & 10.9 & - & 0.034 \\
\hline$\gamma_{2}, \mathbf{k}_{2}$ & 22 & - & 0.415 \\
\hline$\gamma_{3}, \mathbf{k}_{3}$ & 20.1 & - & 0.088 \\
\hline$\gamma_{4}, \mathbf{k}_{4}$ & 5.3 & - & 0.079 \\
\hline
\end{tabular}

The time responses of these optimum design points and obtained results in [8] are compared in Figs. 9 and 10 and their control inputs are illustrated in Fig. 11. Considerably, the SDSAF method generates smaller settling time, and its control input is smoother than the two other methods.

\begin{tabular}{l|c|c|c}
\hline & $\begin{array}{c}\text { FBL } \\
\text { (point C) }\end{array}$ & $\begin{array}{c}\text { DSMC } \\
\text { (point B) }\end{array}$ & $\begin{array}{c}\text { SDSAF } \\
\text { (point A) }\end{array}$ \\
\hline $\boldsymbol{P}_{s}$ & - & 1.98 & 0.91 \\
\hline $\boldsymbol{G}$ & - & 4.39 & 0.77 \\
\hline $\mathbf{C}_{\mathrm{s}}$ & - & - & 0.73 \\
\hline $\mathbf{C}_{\mathrm{f}}$ & - & - & 0.9 \\
\hline $\mathbf{k}_{\mathbf{1}}(\mathbf{0})$ & - & - & 0.52 \\
\hline $\mathbf{k}_{2}(\mathbf{0})$ & - & - & 0.31 \\
\hline $\mathbf{k}_{\mathbf{3}}(\mathbf{0})$ & - & - & 0.7 \\
\hline $\mathbf{k}_{4}(\mathbf{0})$ & - & - & 0.25 \\
& & & \\
\hline
\end{tabular}

\section{Figure 9}

Time responses of the beam angle corresponding to the optimum design points A, B, and C shown in the Pareto fronts and [8]

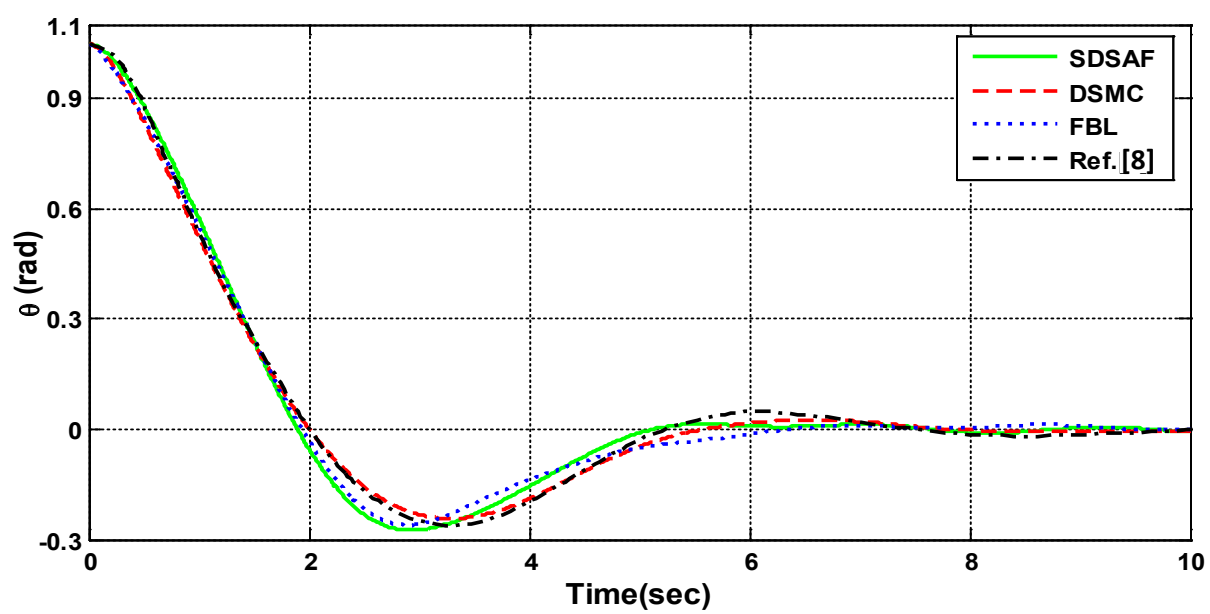


Figure 10

Time responses of the ball position corresponding to the optimum design points A, B, and C shown in the Pareto fronts and [8]

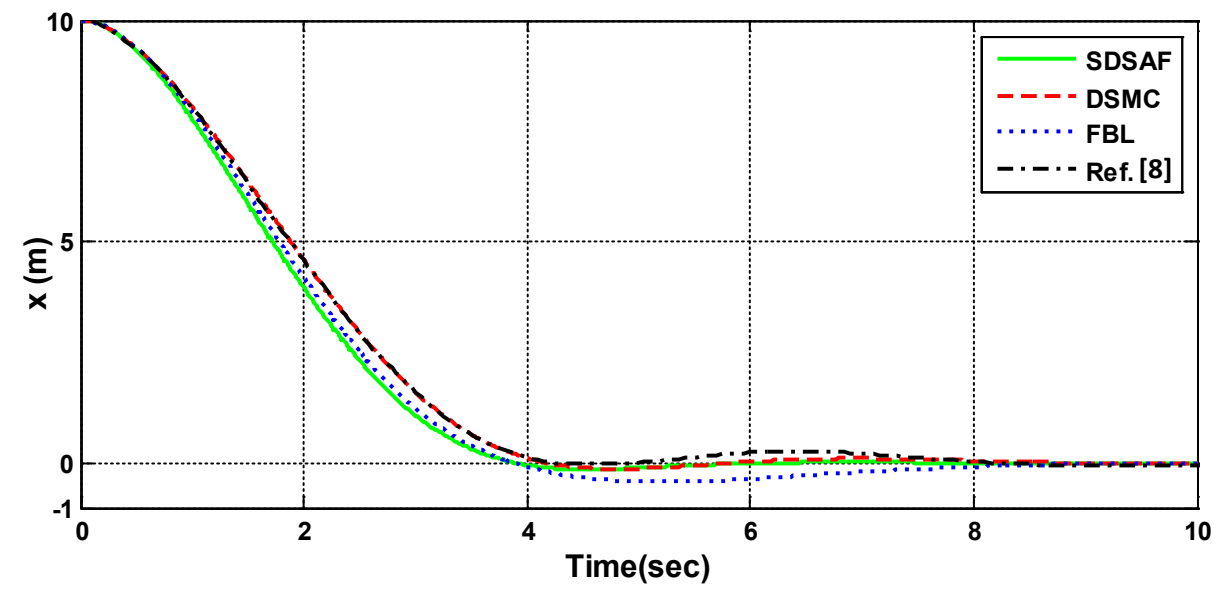

Figure 11

Control input of the ball-beam system corresponding to the optimum design points A, B, and C shown in the Pareto fronts and [8]

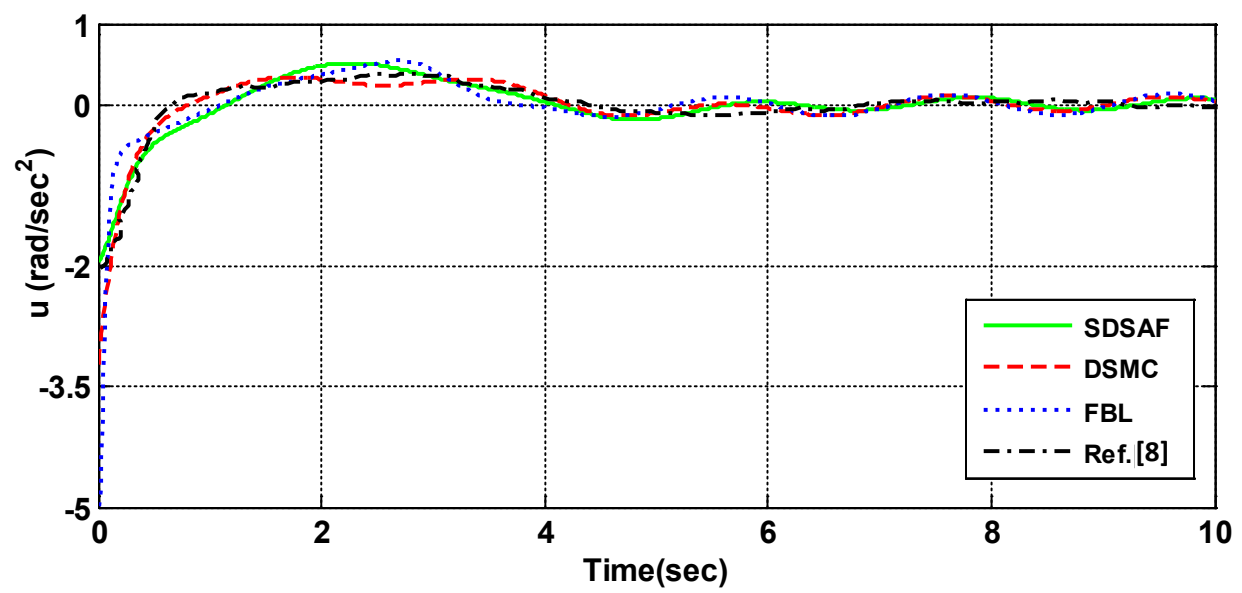

\subsection{Ball-wheel}

The dynamic behavior of the ball-wheel system shown in Fig. 12 can be expressed by the following nonlinear equations:

$$
\dot{x}_{1}(t)=x_{2}(t)
$$

$$
\begin{aligned}
& \dot{x}_{2}(t)=a x_{4}(t)+b \sin x_{1}(t)+c u(t) \\
& \dot{x}_{3}(t)=x_{4}(t) \\
& \dot{x}_{4}(t)=p x_{4}(t)+q \sin x_{1}(t)+r u(t),
\end{aligned}
$$


where $\left[x_{1}(t), x_{2}(t), x_{3}(t), x_{4}(t)\right]=$

$\left[\theta_{1}(t), \dot{\theta}_{1}(t), \theta_{2}(t), \dot{\theta}_{2}(t)\right]$ and:

$a=-\frac{2 r_{w} k_{m}^{2}}{R_{a}\left(7 I_{w}+2 r_{w}^{2} m_{b}\right)\left(r_{b}+r_{w}\right)} b=\frac{8\left(5 I_{w}+2 r_{w}^{2} m_{b}\right)}{\left(7 I_{w}+2 r_{w}^{2} m_{b}\right)\left(r_{b}+r_{w}\right)}$

$c=\frac{2 r_{w} k_{m}}{R_{a}\left(7 I_{w}+2 r_{w}^{2} m_{b}\right)\left(r_{b}+r_{w}\right)} \quad p=\frac{-7 k_{m}^{2}}{R_{a}\left(7 I_{w}+2 r_{w}^{2} m_{b}\right)}$

$q=\frac{2 g r_{w} m_{b}}{\left(7 I_{w}+2 r_{w}^{2} m_{b}\right)} \quad r=\frac{7 k_{m}}{R_{a}\left(7 I_{w}+2 r_{w}^{2} m_{b}\right)}$.

Figure 12

The physical configuration of the ball-wheel system

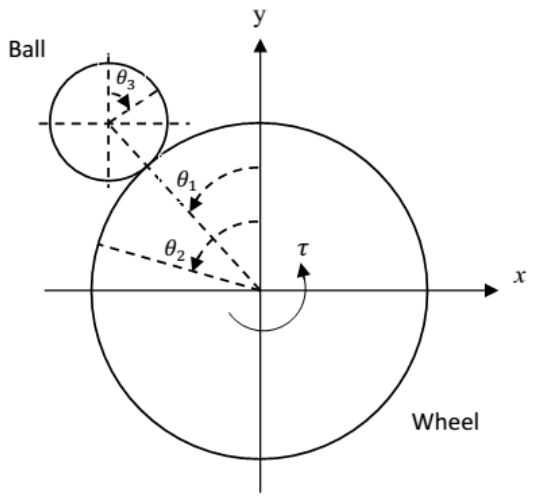

It is noted that $a r=c p$. Initial values are $\theta_{1}(0)=-0.08, \dot{\theta}_{1}(0)=0, \theta_{2}(0)=0.08, \dot{\theta}_{2}(0)=0 . I_{w}, r_{w}, m_{b}, r_{b}$,

$R_{a}$, and $k_{m}$ are inertia moment of the wheel, radius of the wheel, mass of the ball, radius of the ball, motor armature resistance, and motor constant, respectively. The used specifications are as follows: $I_{w}=1.71 \times$ $\left.10^{-3}\right) \mathrm{kg} \cdot \mathrm{m}^{2}, r_{w}=0.075 \mathrm{~m}, \mathrm{~m}_{b}=0.042 \mathrm{~kg}, r_{b}=0.011 \mathrm{~m}, R_{a}=$ $0.6558 \Omega, k_{m}=0.0662 \mathrm{Nm} / \mathrm{A}$. By selecting the sliding surface based on Equations (5) and (6), the decoupled sliding mode controller is defined as Equation (52):

$$
\begin{aligned}
& u_{D S M C}(t)=\frac{1}{c}\left(c_{1}\left(\dot{z}-x_{2}(t)\right)-\right. \\
& \left.\left(a x_{4}(t)+b \sin x_{1}(t)\right)\right)-P_{S} \operatorname{sat}\left(s_{1} G\right) .
\end{aligned}
$$

For implementation of FBL method, the first state is chosen as $\zeta_{1}=\varnothing(\mathbf{x})=r x_{1}(t)-c x_{3}(t)[5]$, then other states would be obtained as follows:

$$
\begin{aligned}
& \dot{\zeta}_{1}=\zeta_{2}=L_{f} \emptyset(\mathbf{x})=r x_{2}(t)-c x_{4}(t) \\
& \dot{\zeta}_{2}=\zeta_{3}=L_{f}^{2} \emptyset(\mathbf{x})=(b r-c q) \sin x_{1}(t) \\
& \dot{\zeta}_{3}=\zeta_{4}=L_{f}^{3} \emptyset(\mathbf{x})=(b r-c q) x_{2}(t) \cos x_{1}(t) \\
& \dot{\zeta}_{4}=L_{f}^{4} \emptyset(\mathbf{x})+L_{g} L_{f}^{3} \emptyset(\mathbf{x}) u(t)= \\
& -(b r-c q) x_{2}^{2}(t) \sin x_{1}(t)+ \\
& (b r-c q)\left(a x_{4}(t)+b \sin x_{1}(t)\right) \cos x_{1}(t)+ \\
& c(b r-c q) \cos x_{1}(t) u(t)
\end{aligned}
$$

\section{Figure 13}

The obtained Pareto fronts by using MATLAB's Toolbox MOGA for FBL, DSMC, and SDSAF for the ball-wheel system

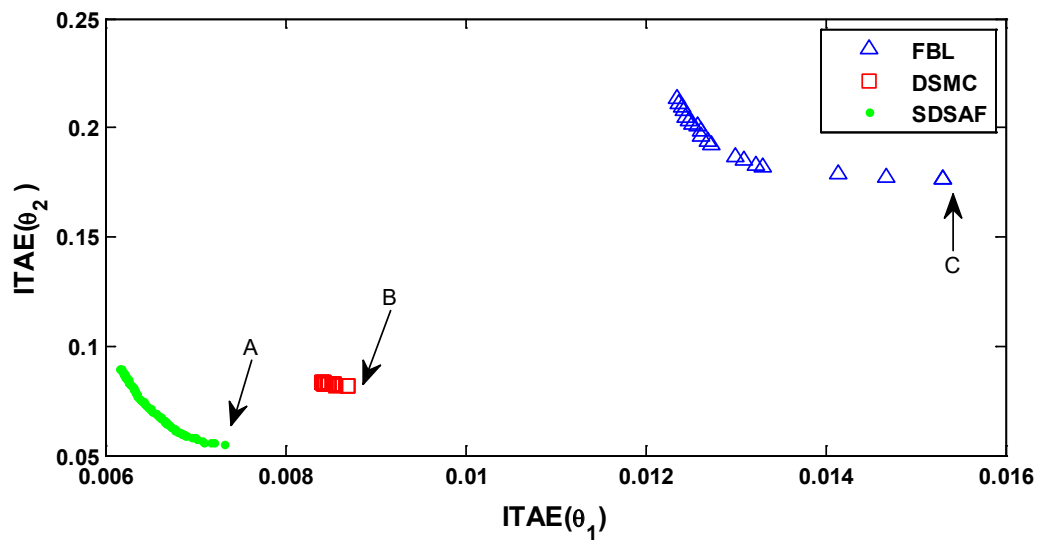


$u_{F B L}(t)=$ $\frac{v-\left(-(b r-c q) x_{2}^{2}(t) \sin x_{1}(t)+(b r-c q)\left(a x_{4}(t)+b \sin x_{1}(t)\right) \cos x_{1}(t)\right)}{c(b r-c q) \cos x_{1}(t)}$.

The Pareto front, which is achieved by applying the multi-objective optimization, is shown in Fig.13, and specifications of designated optimum points are shown in Tables 5 and 6, respectively. These results considerably show that the SDSAF method generates less value of the objective functions. The time responses of these optimum design points and obtained results in [5] are compared in Figs. 14 and 15. Furthermore, their control inputs are illustrated in Fig. 16. As a result, Figs. 4, 5 , and 6 conspicuously show that the SDSAF method exhibits a faster response than the other methods, and the maximum overshoot is lower, too.

\section{Figure 14}

Time responses of the ball angle corresponding to the optimum design points A, B, and C shown in the Pareto front and [5]

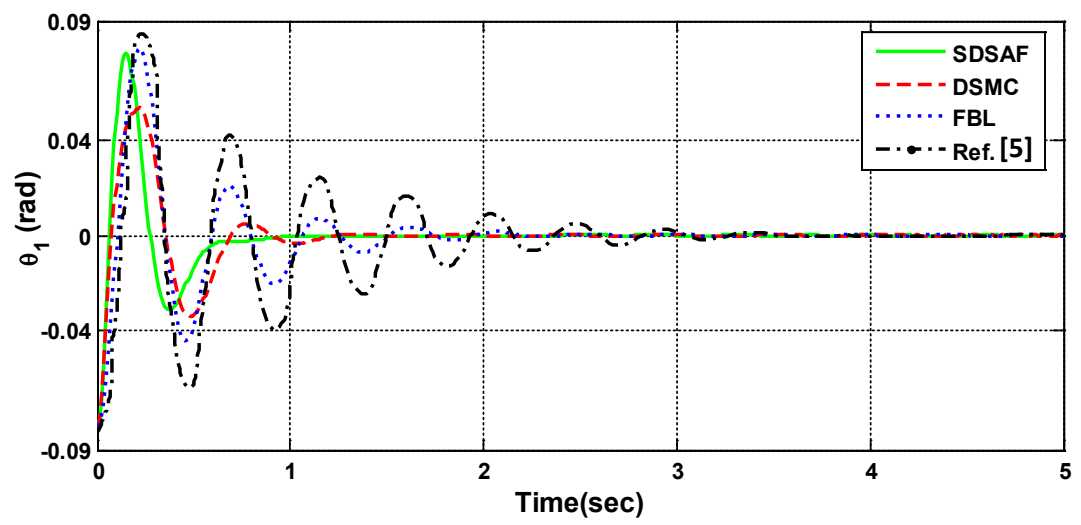

Table 5

Values of optimization constraints and objective functions for the optimum points A, B, and C of Fig. 13

\begin{tabular}{l|c|c|c}
\hline & FBL (point C) & DSMC (point B) & SDSAF (point A) \\
\hline $\mathbf{m}_{\mathbf{u}}$ & 3 & 4 & 3 \\
\hline $\mathbf{s}_{\mathbf{u}}$ & 1 & 2 & 1 \\
\hline $\operatorname{ITAE}\left(\boldsymbol{\theta}_{\mathbf{1}}\right) \times \mathbf{1 0}^{-\mathbf{3}}$ & 16.4 & 6.999 & 4.658 \\
\hline $\operatorname{ITAE}\left(\boldsymbol{\theta}_{\mathbf{2}}\right) \times \mathbf{1 0}^{-3}$ & 139.5 & 58.22 & 29.38 \\
\hline
\end{tabular}

\section{Table 6}

Design variables for the optimum points A, B, and C of Fig. 13

\begin{tabular}{l|c|c|c} 
& FBL (point C) & DSMC (point B) & SDSAF (point A) \\
\hline $\mathbf{c}_{\mathbf{1}}$ & - & 3.96 & 8 \\
\hline $\mathbf{c}_{2}$ & - & 3.23 & 3.73 \\
\hline$\emptyset_{\mathbf{z}}$ & - & 6.94 & 21.69 \\
\hline $\mathbf{Z}_{\text {upper }}$ & - & 0.4 & 0.53 \\
\hline$\gamma_{1}, \mathbf{k}_{1}$ & 2148 & - & 0.74 \\
\hline$\gamma_{2}, \mathbf{k}_{2}$ & 915 & - & 0.888 \\
\hline$\gamma_{3}, \mathbf{k}_{3}$ & 220 & - & 0.116 \\
\hline$\gamma_{4}, \mathbf{k}_{4}$ & 8.79 & - & 0.033 \\
\hline
\end{tabular}

\begin{tabular}{l|c|c|c} 
& FBL (point C) & DSMC (point B) & SDSAF (point A) \\
\hline $\boldsymbol{P}_{s}$ & - & 2.32 & 2.92 \\
\hline $\boldsymbol{G}$ & - & 4.48 & 0.47 \\
\hline $\mathbf{C}_{\mathbf{s}}$ & - & - & 0.42 \\
\hline $\mathbf{C}_{\mathrm{f}}$ & - & - & 1.7 \\
\hline $\mathbf{k}_{1}(\mathbf{0})$ & - & - & 0.78 \\
\hline $\mathbf{k}_{2}(\mathbf{0})$ & - & - & 50.65 \\
\hline $\mathbf{k}_{3}(\mathbf{0})$ & - & - & 0.82 \\
\hline $\mathbf{k}_{\mathbf{4}}(\mathbf{0})$ & - & - & 0.696 \\
\hline
\end{tabular}




\section{Figure 15}

Time responses of the wheel angle corresponding to the optimum design points A, B, and C shown in the Pareto front and [5]

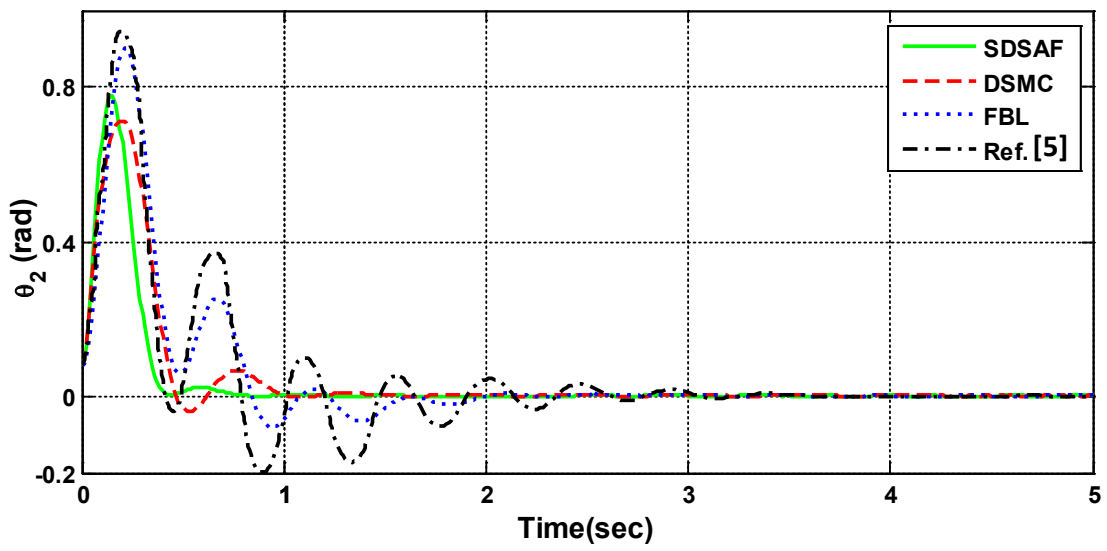

\section{Figure 16}

Control input of the ball-wheel system corresponding to the optimum design points A, B, and C shown in the Pareto fronts and [5]

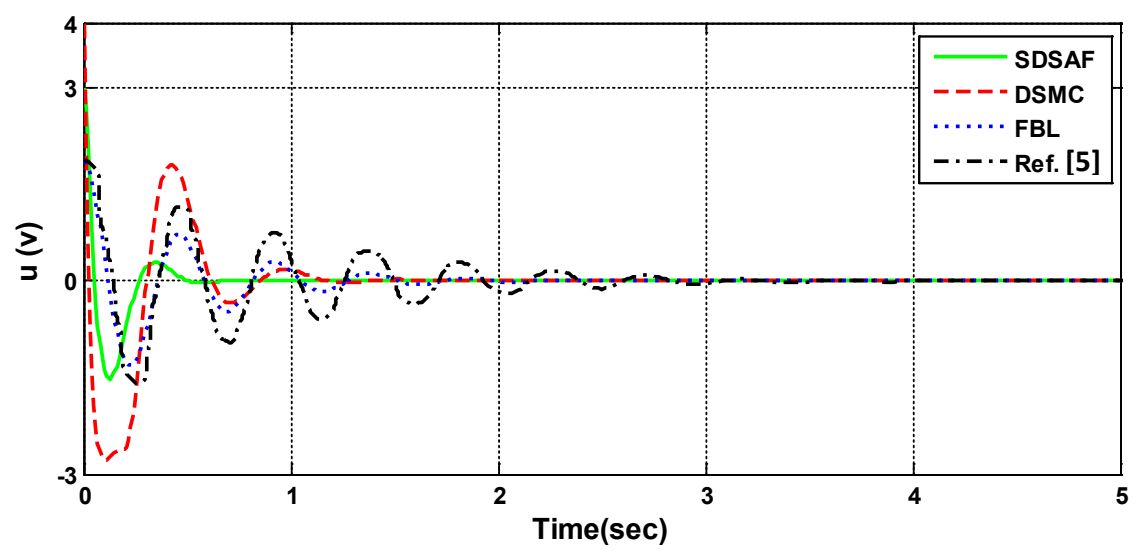

\section{Conclusions}

A hybrid optimal controller based on a combination of robust decoupled sliding mode and adaptive feedback linearization, briefly, called SDSAF, was proposed for a class of fourth order systems. First, the decupled sliding mode control and feedback linearization was implemented simultaneously. Then, summation of the weighting control law was used as a new control effort. Finally, the effective parameters were optimized by the multi-objective genetic algorithm. The simulation results for the cart-pole, ball-beam and ball-wheel systems were provided to show the robustness and effectiveness of the SDSAF method. Simulation results demonstrated that the dynamic responses obtained from the proposed controller are much faster than those obtained from the FBL, DSMC and other approaches considered in literature. In addition, the SDSAF method exhibited lower ITAE values compared with the DSMC and FBL methods. 


\section{References}

1. Andalib Sahnehsaraei, M., Mahmoodabadi, M. J., Bagheri, A. Pareto Optimum Control of a 2-DOF Inverted Pendulum Using Approximate Feedback Linearization and Sliding Mode Control. Transactions of the Institute of Measurement and Control, 2013, 36(4), 496-505. https://doi.org/10.1177/0142331213504946

2. Bayramoglu, H., Komurcugil, H. Nonsingular Decoupled Terminal Sliding-Mode Control for a Class of Fourth-Order Nonlinear Systems. Communication Nonlinear Science and Numerical Simulation, 2013, 18(9), 2527-2539. https://doi.org/10.1016/j.cnsns.2012.11.008

3. Bayramoglu, H., Komurcugil, H. Time-varying Sliding-Coefficient-Based Decoupled Terminal Sliding-Mode Control for a Class of Fourth-order Systems. ISA Transactions, 2014, 53(4), 1044-1053.https://doi. org/10.1016/j.isatra.2014.05.013

4. Hauser, J., Sastry, S., Kokotovic, P. Nonlinear Control via Approximate Input-Output Linearization: The Ball and Beam Example. IEEE Transactions on Automatic Control, 1992, 37(3), 392-398. https://doi. org/10.1109/9.119645

5. Ho, M. T., Tu, Y. W., Lin, H. S. Controlling a Ball and Wheel System Using Full-State-Feedback Linearization. IEEE Control Systems, 2009, 29(5), 93-101. https://doi.org/10.1109/MCS.2009.934085

6. Hung, L. C., Chung, H. Y. Decoupled Sliding-Mode with Fuzzy-Neural Network Controller for Nonlinear Systems. International Journal of Approximate Reasoning, 2007, 46(1), 74-97. https://doi.org/10.1016/j.jjar.2006.08.002

7. Khalil, H. K. Nonlinear System. New Jersey, Prentice Hall, 1996.

8. Lo, J., Kuo, Y. Decoupled Fuzzy Sliding-Mode Control. IEEE Transactions on Fuzzy Systems, 1998, 6(3), 426-435. http://ieeexplore.ieee.org/document/705510/. https://doi. org/10.1109/91.705510
9. Mahmoodabadi, M. J., Arabani Mostaghim, S., Bagheri, A., Nariman-zadeh, N. Pareto Optimal Design of the Decoupled Sliding Mode Controller for an Inverted Pendulum System and Its Stability Simulation via Java Programming. Mathematical and Computer Modelling, 2013, 57(5-6), 1070-1082. https://doi.org/10.1016/j. mcm.2012.06.027

10. Mahmoodabadi, M. J., Momennejad, S., Bagheri, A. Online Optimal Decoupled Sliding Mode Control Based on Moving Least Squares and Particle Swarm Optimization. Information Sciences, 2014, 268, 342-356. https:// doi.org/10.1016/j.ins.2014.01.027

11. Mahmoodabadi, M. J., Taherkhorsandi, M., Bagheri, A. Optimal Robust Sliding Mode Tracking Control of a Biped Robot Based on Ingenious Multi-Objective PSO. Neurocomputing, 2014, 124, 194-209. https://doi. org/10.1016/j.neucom.2013.07.009

12. Mahmoodabadi, M. J., Taherkhorsandi, M., Talebipour, M., Castillo-Villar, K. K. Adaptive Robust PID Control Subject to Supervisory Decoupled Sliding Mode Control Based upon Genetic Algorithm Optimization. Transactions of the Institute of Measurement and Control, 2015, 37(4), 505-514. https://doi. org/10.117r/0142331214543295

13. Slotine, J. J. E., Li, W. Applied Nonlinear Control. New Jersey, Prentice Hall, 1991.

14. Taherkhorsandi, M., Mahmoodabadi, M. J., Talebipour, M., Castillo-Villar, K. K. Pareto Design of an Adaptive Robust Hybrid of PID and Sliding Control for a Biped Robot via Genetic Algorithm Optimization. Nonlinear Dynamics, 2015, 79(1), 251-263. https://doi. org/10.1007/s11071-014-1661-1

15. Utkin, V., Guldner, J., Shi, J. Sliding Mode Control in Electromechanical Systems. Boca Raton, FL: CRC Press, 1999. 